\title{
A Method to Design an Integrated MO Head Using a Diffraction Calculation in Biaxial Crystals
}

\author{
Noriaki Nishi, Kiyoshi Toyota, Kimihiro Saito* \\ Computer Peripherals \& Components Company, Sony Corporation, 6-7-35, Kitashinagawa, Shinagawa-ku, Tokyo 141-0001, Japan \\ *Giga Byte Laboratories, Sony Corporation, 6-7-35, Kitashinagawa, Shinagawa-ku, Tokyo 141-0001, Japan
}

\section{Introduction :}

MiniDisc (MD) has become the most popular rewritable optical disc. In the MD system, a $64 \mathrm{~mm}$-diameter disc in a cartridge can record and play back $74 \mathrm{~min}$. of compressed audio data or store $140 \mathrm{MB}$ of user data, using magnetooptical (MO) technology. In the MD market, there is a great demand for smaller, lighter and more stable optical heads with lower material and production costs. As an approach to satisfy these requirements, we have presented a new integrated optical device which we call MiniDisc Laser Coupler (MD-LC)[1]. In MD-LC, we integrate all functions necessary for MD optical heads into one small chip. We have reported its simple configuration and some experimental results which show its ability to detect signals with high quality and high stability against an environmental change. In this paper, we describe how we designed MD-LC using a new method to make diffraction calculations in biaxial crystals.

\section{MiniDisc Laser Coupler :}

2-1 Reduction of optical components : Fig.1 shows the schematic diagram of the new optical head employing MD-iC. In our device, all optical elements except the lens are replaced with only one non-laminated micro-prism made out of a birefringent biaxial crystal, KTP (KTiOPO4), for MO signal detection. Our device can also detect all servo signals, such as focusing and tracking error signals, an ADIP (ADdress In Pregroove) signal and a FAPC (Front Auto Power Contr, 1) signal for auto laser power control, without additic al optical components. MD-LC contributes to the reduction of optical components in the MD optical head, from 9 in tie conventional head, to only 2.

2.2 Optical paths in the birefringent KTP micro-prism : The light frum the laser diode is reflected by a beam splitter on the slope of the KTP micro-prism and focused on the disc. The reflected light from the disc which passes through the beam splitter is further divided by a half mirror located on the bottom of the prism. The light which passes the half mirror is detected by the photo detector " $A$ " shown in Fig.1, and the light reflected by the mirror is again reflected by a high reflection film on the upper surface of the prism. Finally the photo detectors " $I$ " and " $J$ " detect the light from the disc. The MO signal is obtained from the difference "I" - "J". In our design, there can be 8 spots around the detectors " $I$ " and " $J$ ", because of one refraction on the slope and two internal reflections. (At each interface, each beam is devided in two.) But we succeeded in reducing the number of spots from 8 to 2 by properly orienting the optical axis of the KTP crystal. Details about the method for reducing the number of spots will be shown in section 4.

\section{A diffraction calculation in biaxial crystals :}

In the process of manufacturing Laser Coupler, the laser diode, the micro-prism and the photo detectors are located, with no adjustment, on a common silicon substrate in positions determined in advance, in order to achieve higher processing ability and higher stability. Therefore, we have to know the exact distribution of light intensity on the detector plane, which can be obtained through diffraction calculations, and we use this information to optimize the positions and shapes of the detectors. We usually ray-trace and make diffraction calculations based on the wave front normal vector $\mathbf{k}$, because in isotropic media like glass, the Poynting vector $\mathbf{S}$ is parallel to $\mathbf{k}$, and $\mathbf{k}$ obeys Snell's law. Here Poynting vector gives the direction and magnitude of the energy flow. In birefringent crystals, $\mathbf{S}$ is not parallel to $\mathbf{k}$ any more (which is called "walk-off") so that $\mathbf{S}$ does not obey Snell's law, and hence existing methods cannot be applied to optical systems employing birefringent crystals. It was necessary for us to develop a method to ray-trace and to make diffraction calculations in crystals, especially in biaxial crystals. 
Procedures: First of all, we performed a ray-tracing, considering "walk-off " effects, along the direction of the Poynting vector $\mathbf{S}$. This ray-tracing method was used for calculating not only spot diagrams but also optical path distances (OPD) necessary for diffraction calculations. Then, a pupil function described with Jones vectors was calculated. Diffraction by the disc and the variation of polarization directions were taken into account. After being multiplied by aberration and transmittance, the pupil function was Fourier-transformed into the distribution of light intensity on the detector plane. In our calculation, "aberration" was obtained through considering OPD based on Poynting vector and optical phase difference generated by transmissions and reflections on surfaces with multi-layered thin films on them. In order to obtain exact transmittance and reflectance, we also calculate two eigen-polarizations and amplitudes of corresponding $\mathbf{E}$ vectors, before and after a refraction or a reflection on a surface of crystal with multilayered thin films on it. This calculation is made faithfully according to the electromagnetic boundary conditions given by Maxwell's Equations.

\section{Setup of crystal axis :}

Fig. 2 shows the calculated results of optical paths (Fig.2a), spot diagrams (Fig.2b) and diffraction patterns (Fig.2c) on detectors in our system. One refraction and two reflections generate 8 spots on detectors in Fig. $2 \mathrm{~b}$. Two of these spots are perfectly overlapped so that only 6 spots can be seen. We also calculated aberration and transmittance of every optical path and then computed the distributions of light intensity for each of the eight spots, because no information about the distributions was given by spot diagrams. The calculated distributions are shown in Fig. $2 \mathrm{c}$ with the detector patterns. Only two spots can be seen in Fig.2c. This means that unnecessary beam separations, which are normally caused by two internal reflections, has been reduced owing to our optimum setup of crystal axis. The adequacy of our arrangement of photo detectors have been already verified from the results in our previous paper[1].

\section{Conclusion:}

We developed a method to ray-trace and make diffraction calculations in biaxial crystals and applied it to the development of the new integrated laser coupler for MD systems which we call MD-LC. We have successfully reduced beam separation caused by internal reflections in crystal, because of our optimum setup of the crystal axis. We have optimized the arrangement of detectors utilizing this method. Using MD-LC we can make small, light and stable optical heads for MD.

\section{Reference:}

[1] N.Nishi et al, ODS 98 Technical Digest, pp52-54

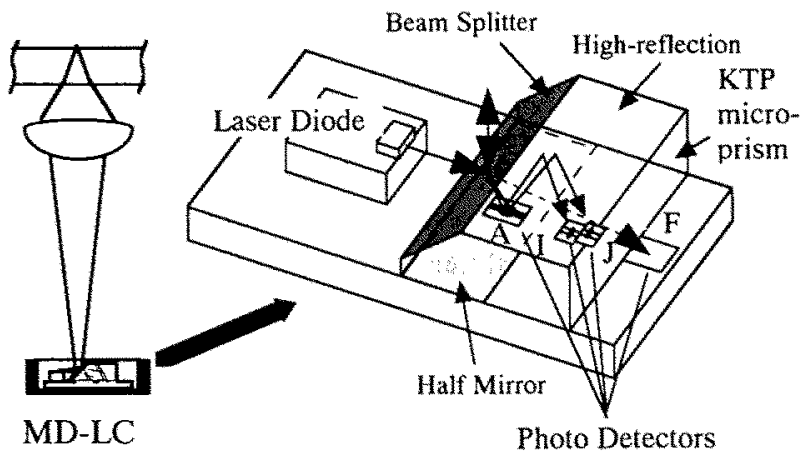

MD-LC

Package Size $: 7.5(\mathrm{~L})^{*} 6.5(\mathrm{~W}) * 1.8(\mathrm{H}) \mathrm{mm}^{3}$

Fig.1 The schematic diagram of MD-LC

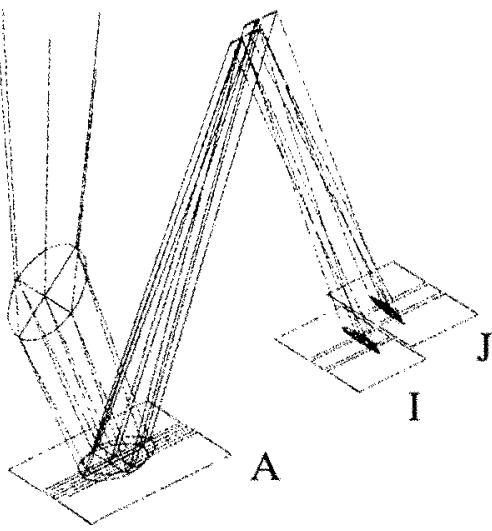

(a) Optical paths

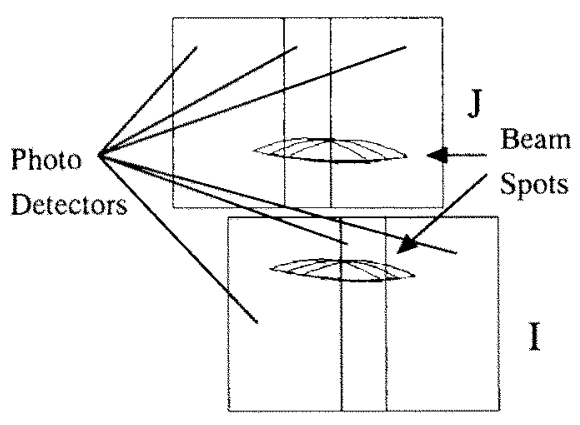

(b) Spot diagrams (boundary plot)

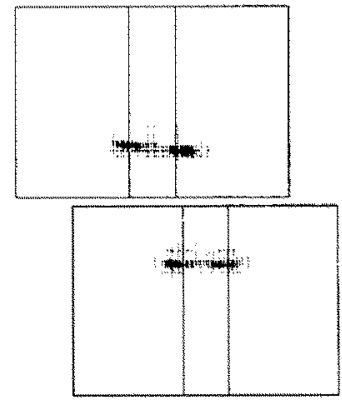

(c) Diffraction patterns

Fig.2 The calculated results of optical paths, spot diagrams and diffraction patterns in our system 